\title{
VARIATION OF HAEMATOLOGICAL INDICES IN MALARIA FEBRILE ILLNESSES
}

\author{
Avinash Gupta1, Pushpraj Singh Baghel2, Ankana Thakur³, Vikash Jain ${ }^{4}$, Neeraj Jain ${ }^{5}$ \\ ${ }^{1}$ Senior Resident, Department of Pathology, NSCB Medical College, Jabalpur. \\ ${ }^{2}$ Assistant Professor, Department of Pathology, NSCB Medical College, Jabalpur. \\ ${ }^{3}$ Senior Resident, Department of Pathology, NSCB Medical College, Jabalpur. \\ ${ }^{4}$ Senior Resident, Department of Pathology, NSCB Medical College, Jabalpur. \\ ${ }_{5}^{5}$ Assistant Professor, Department of Medicine, NSCB Medical College, Jabalpur.
}

\section{ABSTRACT}

\section{BACKGROUND}

Malaria is an epidemiological problem at the global level caused by the Plasmodium species. In Indian subcontinent, it is a major cause of mortality and considered as the main differential diagnosis of acute febrile illness. The parasite mainly infects the erythrocytes and causes various haematological abnormalities like anaemia, thrombocytopenia and Disseminated Intravascular Coagulation (DIC).

AIM

To evaluate the diagnostic relevance of haematological parameters in predicting malaria.

\section{SETTINGS AND DESIGN}

Hospital based prospective cohort study.

\section{MATERIALS AND METHODS}

Blood samples from febrile patients were tested for malaria by the peripheral blood smear study; 100 smear positive malaria cases detected at Central Laboratory Department of Pathology, N.S.C.B. Medical College, Jabalpur, MP, during the period of September 2011 to October 2012, were selected and studied for haematological changes using an automated cell counter. The patients already taking antimalarial drugs were excluded.

\section{RESULTS}

Out of the 100 smear positive malaria cases detected, $63 \%$ had P. vivax infection, while $35 \%$ were infected with P. Falciparum and $2 \%$ had mixed infection. Thrombocytopenia (platelet count less than $150,000 / \mathrm{mm} 3$ ) was seen in $88 \%$ patients and the degree of parasitaemia correlated with extent of thrombocytopenia, the Correlation Co-efficient ( $\mathrm{r}$ ) was $-0.46(\mathrm{P}<0.0001)$, which is highly significant. Anaemia $(\mathrm{Hb}<11 \mathrm{gm} \%)$ was seen in $81 \%$ of the cases. Degree of parasitaemia correlated with the degree of anaemia and the Correlation Co-efficient ( $\mathrm{r}$ ) was- $0.50 \mathrm{P}<0.0001$ ), which is highly significant. Changes in RDW, MPV and leukocyte count were also predictive.

\section{CONCLUSION}

The conclusion of this study is that the presence of thrombocytopenia and anaemia in a patient with acute febrile illness increases the probability of malarial infection and they are more pronounced in P. falciparum infection. Degree of anaemia, RDW and thrombocytopenia usually correlates with parasite load.

\section{KEYWORDS}

Malaria, Thrombocytopenia, Anaemia, Fever, Haematological Changes.

HOW TO CITE THIS ARTICLE: Gupta A, Baghel PS, Thakur A, et al. Variation of haematological indices in malaria febrile illnesses. J. Evolution Med. Dent. Sci. 2016;5(30):1548-1552, DOI: 10.14260/jemds/2016/364

\section{INTRODUCTION}

Malaria is a major health problem in the tropics with 300-500 million cases of malaria occurring annually and an estimated 1.1-2.7 million deaths each year as a result of severe malaria.[1] Malaria is an acute, recurrent and sometimes chronic vectorborne protozoan disease, which has worldwide distribution in tropical and subtropical regions.

Financial or Other, Competing Interest: None.

Submission 22-02-2016, Peer Review 18-03-2016,

Acceptance 24-03-2016, Published 13-04-2016.

Corresponding Author:

Dr. Pushpraj Singh Baghel,

F-44, Doctors Colony,

NSCB Medical College, Garha,

Jabalpur, Madhya Pradesh.

E-mail: drpushprajsingh@gmail.com

DOI: $10.14260 /$ jemds $/ 2016 / 364$
The main areas where disease predominates are the rural and remote areas, where prompt treatment is not available or not detected in time. Malaria causing plasmodia is a parasite of blood and hence induces various haematological changes; some of these changes may be species specific.

\section{Red Blood Cell Changes in Malaria}

Anaemia is one of the most common haematological alterations in Malaria. One arbitrary definition of malarial anaemia in such a setting would be haemoglobin less than 8 $\mathrm{g} / \mathrm{dL}$, which is equivalent to a haematocrit of less than $24 \%$ in a parasitaemic individual. Definition of Severe Malarial Anaemia (SMA) is less problematic. The World Health Organization has defined SMA as haemoglobin less than $5 \mathrm{~g} / \mathrm{dL}$ or a haematocrit less than $15 \%$ seen in the context of malaria, but without specifying parasitaemia. Blood indices (MCV, 
$\mathrm{MCH}, \mathrm{MCHC}$ and RDW) may also change in various types of malaria. Most of the times ESR is increased, whereas reticulocyte count may be decreased or normal.

\section{Leukocyte Variation in Malaria}

Changes in the total WBC in malaria are generally reflected by changes in the neutrophil counts. Although, neutropenia due to increased neutrophil margination is said to be common in acute malaria, in some cases leukocytosis may be seen. More recent literature shows that lymphopenia, which is sometimes profound is a common finding in acute malaria. Monocytosis is also a feature of malaria.

\section{Variation in Platelet Parameter}

Thrombocytopenia is a common and early sign of malarial infection. Platelet abnormalities are both qualitative as well as quantitative. Various platelet indices also show changes like plateletcrit value and platelet distribution width may be decreased with normal or low mean platelet volume.

WHO recommends parasitological confirmation of malaria through quality assured diagnosis in all settings before treatment is started. Treatment solely on the basis of clinical suspicion should only be considered when a parasitological diagnosis is not accessible, i.e. not available within two hours of patients presenting for treatment. As the target of malaria parasite is RBC, present study aims to detect if certain haematological indices would increase the probability of malaria in patients with acute febrile illness. Presence of such an indicator may heighten the suspicion for malaria, prompting a more diligent search for the malaria parasite and prompt institution of specific therapy.

\section{MATERIAL AND METHOD}

This is a prospective study carried out from September 2011 to October 2012. The patient who came with complaint of fever and positive for malaria on peripheral smear was included in the study. All 100 smear positive malaria cases detected at Central Laboratory were selected and studied for haematological changes. Blood sample for haematological study was taken before starting anti-malarial drugs in all these cases. Venous blood was collected in EDTA Vial and 3.8\% sodium citrate vial (for ESR estimation) Haemoglobin ( $\mathrm{Hb}$ ), Haematocrit (HCT), RBC indices like MCV (Mean Corpuscular Volume), MCH (Mean Corpuscular Haemoglobin), MCHC (Mean Corpuscular Haemoglobin Concentration), Red Cell Distribution Width (RDW), total leukocyte count, absolute leukocyte counts, platelet count, Plateletcrit (PCT), Mean Platelet Volume (MPV), and Platelet Distribution Width (PDW) were measured using EDTA blood sample in automated haematology analyzer.

We used Erma PCE-210, which is a fully automated haematology analyzer marketed by Erma Inc. Thin blood smears were prepared using fresh blood sample stained with Leishman stain and studied for blood picture, differential leukocyte count, species identification and for estimation of parasitaemia. Percentage of parasitaemia in thin blood smears was estimated by counting the number of infected RBC's per 100 RBC's.

\section{OBSERVATION}

Out of the total 100 cases studied numbers of vivax cases were 63 , falciparum were 35 and mixed cases were 2 . More than one-third of the cases seen were of falciparum (35\%), which shows increasing trend of falciparum malaria cases. Most of the cases (69\%) were in the young adults between 15-45 years' age group with significant number of cases in children (20\%). In this study falciparum cases were equal in both sexes, while vivax infections were found more in males than females and mixed infection was distributed equally.

Parasitaemia of $1-5 \%$ was seen in a maximum number of cases (61\%); 31 cases of the falciparum infections (88.57\%), showed increased parasite count $(1-5 \%$ or $>5 \%$ range); 9 cases of falciparum malaria (25.71\%) showed high parasite count ( $>5 \%$ ) compared to 5 cases of vivax malaria (7.93\%).

\section{Red Cell Parameters}

Majority of the patients had either mild (39\%) or moderate degree (25\%) of anaemia. Hb concentration $<5 \mathrm{gm} \%$ was seen in total 17 cases, which was proportionately more in cases of falciparum malaria (22.8\%) compared to vivax malaria cases (12.6\%). Hb concentration $>11$ gm $\%$ was seen in total 19 cases, which was proportionately more in cases of vivax malaria $(22.2 \%)$ compared to falciparum malaria cases (14.2\%).

\section{Regression model for prediction of HB Concentration with Parasitaemia}

Regression model for prediction of parasite density we have included RDW, Platelets count and Haemoglobin as predictors in model. Model shows that $\mathrm{R}^{2}=0.46$ and the equation found that parasite density will be 0.895 (Constant)+0.21 RDW0.000012 Platelets count -0.1205 Haemoglobin.

\begin{tabular}{|c|c|c|c|c|}
\hline \multirow[b]{2}{*}{$\begin{array}{c}\text { Parasitaemia } \\
\text { (\%) }\end{array}$} & \multicolumn{4}{|c|}{$\mathrm{Hb}(\mathrm{gm} / \mathrm{dL})$} \\
\hline & $\begin{array}{c}<5 \\
(n=17)\end{array}$ & $\begin{array}{c}5-<8 \\
(n=25)\end{array}$ & $\begin{array}{c}8-1 \\
(n=39)\end{array}$ & $\begin{array}{c}>11 \\
(n=19)\end{array}$ \\
\hline$<1(n=25)$ & 02 & 06 & 06 & 11 \\
\hline $1-5(n=61)$ & 09 & 14 & 30 & 08 \\
\hline$>5(n=14)$ & 06 & 05 & 03 & 00 \\
\hline
\end{tabular}

Severe reduction in $\mathrm{Hb}$ concentration $(<5 \mathrm{gm} / \mathrm{dL})$ was seen in 2 cases of low parasite load $(<1 \%)$ and most cases (15) had parasite load of more than $1 \%$. Normal $\mathrm{Hb}$ concentration $(>11)$ was seen in 19 cases of which low parasite load $(<1 \%)$ accounted for 11 cases and medium load (1-5\%) for 08 cases. Degree of anaemia was correlated with parasite load (Higher the parasite load, more severe the degree of anaemia).

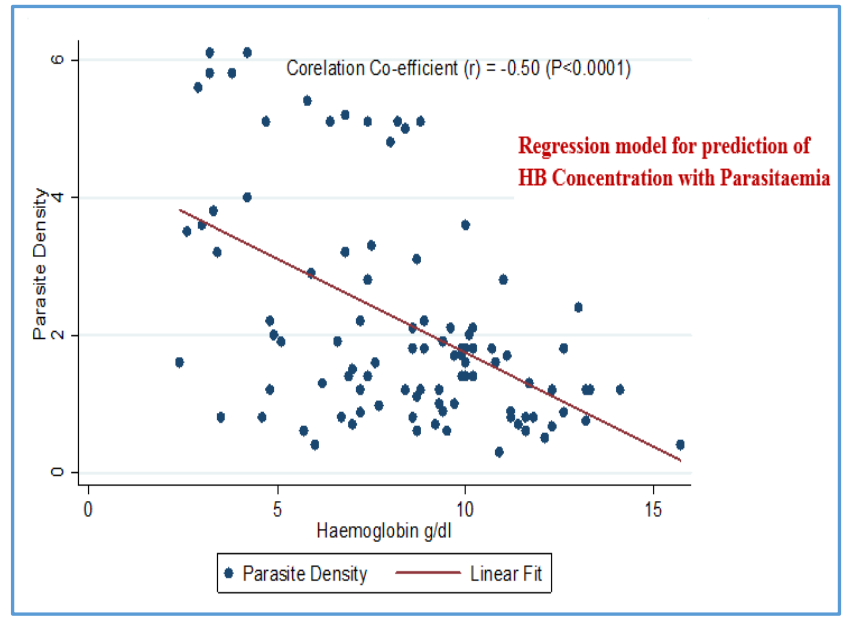


Analysis of correlation coefficient revealed that there was an inverse association between haemoglobin and parasite density $(r=-0.50)$, which is highly significant statistically $(\mathrm{P}<0.0001)$. Double ' $\mathrm{t}$ ' test for correlation of Haemoglobin and $\%$ Parasitaemia (Median=1.65) was also applied and it was highly significant (Double ' $\mathrm{t}$ ' test $(|\mathrm{T}|>|\mathrm{t}|)=0.0010)$.

Severe reduction in $\mathrm{RBC}$ count $(<3$ million $/ \mu \mathrm{L})$ was seen in $38 \%$ of total cases; it was comparatively more common in falciparum malaria (40\%) than to vivax malaria (36.5\%). Haematocrit values of less than 20 were seen more commonly in falciparum infection (25.7\%) compared to vivax malaria cases $(19.04 \%)$. One case of mixed infection showed haematocrit of less than 20. Majority of the patients (76\%) had MCV between $80-100 \mathrm{fL}$. MCV $<80 \mathrm{fL}$ was seen in total 17 cases, which was proportionately more in cases of falciparum malaria (25.71\%) compared to vivax malaria cases (12.69\%). MCV>100 was seen in total 7 cases, which was proportionately more in cases of vivax malaria (6.3\%) compared to falciparum malaria cases (5.7\%). Reduced $\mathrm{MCH}$ and $\mathrm{MCHC}$ values were more commonly seen in P. falciparum malaria cases compared to vivax malaria cases.

RDW was more than $15 \%$ in 72 cases including 9 cases with RDW more than $20 \%$. RDW values of more than 15 were seen more commonly in falciparum infection (77.1\%) compared to vivax malaria cases $(69.8 \%)$.

\section{Regression Model for Prediction of RDW with} Parasitaemia

\begin{tabular}{|c|c|c|c|}
\hline \multirow{2}{*}{$\begin{array}{c}\text { Parasitaemia } \\
(\%)\end{array}$} & $\begin{array}{c}<\mathbf{1 5} \\
(\mathbf{n = 2 6 )}\end{array}$ & $\begin{array}{c}\mathbf{1 5 - 2 0} \\
(\mathbf{n = 6 5})\end{array}$ & $\begin{array}{c}\mathbf{> 2 0} \\
(\mathbf{n = 0 9})\end{array}$ \\
\cline { 2 - 4 } & 09 & 16 & 00 \\
\hline$<1(\mathrm{n}=25)$ & 17 & 38 & 06 \\
\hline $1-5(\mathrm{n}=61)$ & 00 & 11 & 03 \\
\hline$>5(\mathrm{n}=14)$ & \multicolumn{3}{|c|}{ Table 2: $\boldsymbol{R D W}$ with Parasitaemia } \\
\hline \multicolumn{4}{|c}{} \\
\hline
\end{tabular}

High parasitaemia was associated with increased RDW ( $>15 \%$ ). Degree of parasitaemia was positively correlated with extent of increased RDW. The correlation coefficient $(r)=0.46$, which is highly significant statistically $(\mathrm{P}<0.0001)$.

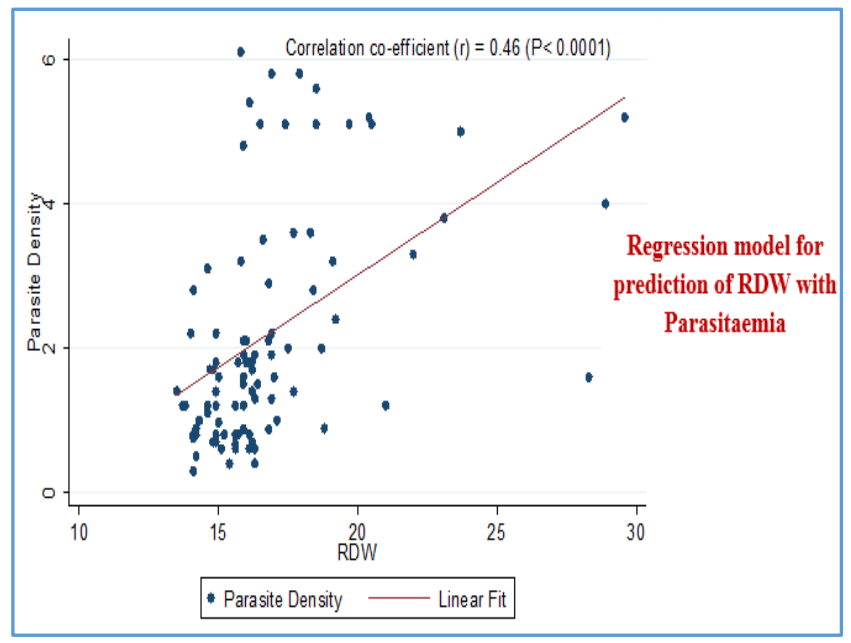

Double ' $\mathrm{t}$ ' test for correlation of RDW and \% Parasitaemia (Median=1.65) was also applied and it was highly significant (Double ' $\mathrm{t}$ ' test $(|\mathrm{T}|>|\mathrm{t}|)=0.0011$ ).

Reticulocyte count was more than $2 \%$ in 46 cases. Increased reticulocyte count was seen mostly in P. falciparum infections (54\%) compared to P. vivax infections (41.2\%).

\section{WBC Parameters}

Majority of the patients had normal WBC count (72\%). Reduced counts were seen in 19 cases and increased counts in 9 cases. Decreased leukocyte count was seen mostly in P. falciparum infections (31.4\%) compared to P. vivax infections (12.6\%). Double ' $\mathrm{t}$ ' test for correlation of TLC and \% Parasitaemia (median=1.65) was done and it was highly significant (Double ' $\mathrm{t}$ ' test $(|\mathrm{T}|>|\mathrm{t}|)=0.0076)$. Increased neutrophil count was present in 10 cases, out of these 10 cases 8 cases were of vivax infection. Reduced neutrophil count was present in 7 cases, 5 cases out of these 7 cases were of falciparum malaria. Eosinophilia was present in 5 cases, out of these 5 cases 4 cases were of vivax infection. Lymphocytosis and monocytosis was seen mainly with falciparum infection. Double ' $t$ ' test for correlation of monocyte and \% Parasitaemia (Median=1.65) was also applied and it was significant (Double ' $\mathrm{t}$ ' test $(|\mathrm{T}|>|\mathrm{t}|)=0.0376)$.

\section{Platelet Parameters}

Decreased platelet counts were a constant feature of both types of malaria with 88 of cases showing platelets less than 1.5 lacs. Severe platelet reduction $(<50,000)$ was seen in 21 cases; it was more common in falciparum (40\%) as compared to vivax case $(11.1 \%)$. MPV values above $12 \mathrm{fL}$ were seen in 21 cases; $25.71 \%$ cases of falciparum showed MPV greater than $12 \mathrm{fL}$ as compared to $19.04 \%$ of cases of vivax malaria. Double ' $\mathrm{t}$ ' test for correlation of MPV and \% Parasitaemia (Median=1.65) was also applied and it was highly significant (Double ' $\mathrm{t}$ ' test $(|\mathrm{T}|>|\mathrm{t}|)=0.0005)$. Plateletcrit values were decreased $(<0.13 \%)$ in most of the cases $(70)$ with almost equal distribution in both falciparum and vivax cases. Platelet Distribution Width values were decreased $(<11.5 \%) 20$ cases, were increased (>14.5\%) in 42 cases.

Regression Model for Prediction of Platelet Count with Parasitaemia

\begin{tabular}{|c|c|c|c|}
\hline \multirow[b]{2}{*}{$\begin{array}{c}\text { Parasitaemia } \\
(\%)\end{array}$} & \multicolumn{3}{|c|}{ Platelet Count $/ \mathrm{mm}^{3}$} \\
\hline & $\begin{array}{c}<50,000 \\
(n=21)\end{array}$ & $\begin{array}{c}50,000-1.5 \text { lac } \\
(n=67)\end{array}$ & $\begin{array}{l}>1.5 \text { lac } \\
(n=12)\end{array}$ \\
\hline$<1(n=25)$ & 01 & 20 & 04 \\
\hline $1-5(n=61)$ & 09 & 45 & 07 \\
\hline$>5(n=14)$ & 11 & 02 & 01 \\
\hline
\end{tabular}

High parasitaemia was associated with marked thrombocytopenia in all except one case. Degree of parasitaemia was correlated with extent of thrombocytopenia. Analysis revealed that coefficient of correlation (r) is -0.46 , which is highly significant statistically $(\mathrm{P}<0.0001)$. 


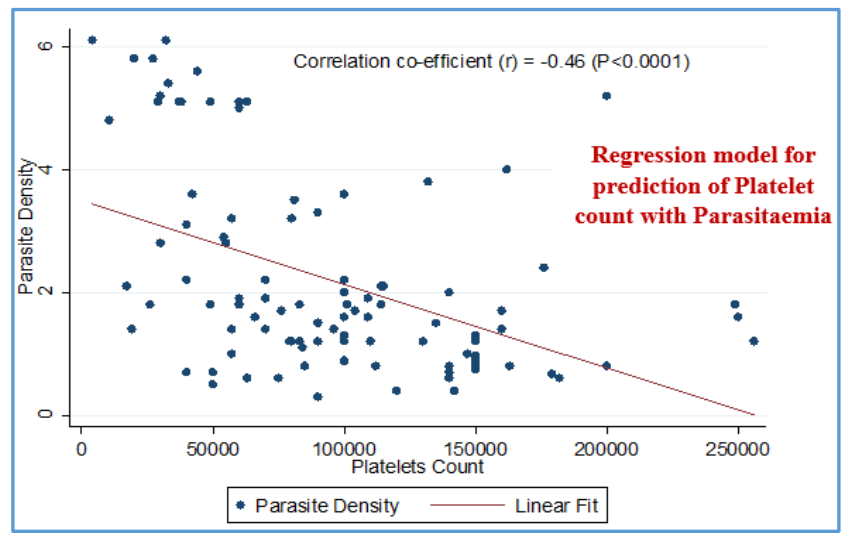

Double ' $\mathrm{t}$ ' test for correlation of Platelet Count and \% Parasitaemia (Median=1.65) was also applied and it was highly significant (Double ' $\mathrm{t}$ ' test $(|\mathrm{T}|>|\mathrm{t}|)=0.0001$ ).

\section{DISCUSSION}

Despite numerous advances in diagnostic methods and treatment modalities, the worldwide incidence of malaria is still about 300-500 million cases annually with 1.1 to 2.7 million deaths.[2] Malaria causes numerous haematological alterations, of which anaemia and thrombocytopenia are the most important. In India where the population has already reduced haemoglobin concentration due to inadequate dietary intake, burden of other infections especially in children, malaria adds to the already fragile health status of the population. The numbers of vivax cases seen were $63 \%$, falciparum $35 \%$ and two mixed infections. Similar results were seen by Erhart et al.[2] Jadhav et al.[3] and Sevenson et al..[4] The mean age group of the present study is 27.4, while Erhart et al.[2] reported a mean age of 28 . Present study had $64 \%$ males similar to other studies with comparable results include Erhart et al.[2] with $69 \%$ males. In the present study, anaemia $(<11 \mathrm{gm} \%)$ was seen in $81 \%$ of the cases. In other studies carried out by Sharma et al.[5] had anaemia in $86.7 \%$ of the cases, while in a study conducted by Biswas et al.[6] showed anaemia in $94.4 \%$ of the cases. In our study, most commonly RBC's were normocytic normochromic (50\%), followed by microcytic hypochromic (46\%). Similar results were found in the study conducted by Layla A M et al. and Facer et al.[7] In our study, RDW was $>15 \%$ in 72 cases including 9 cases with RDW more than 20\%. The Correlation Coefficient (r) was 0.46 $(\mathrm{P}<0.0001)$, which is highly significant and shows that increase in the red cell distribution width is one of the common haematological parameter for the prediction of malaria cases and to assess the parasite load in malaria cases; Double ' $\mathrm{t}$ ' test for correlation of RDW and \% parasitaemia was done and it was highly significant (Double ' $\mathrm{t}$ ' test $(|\mathrm{T}|>|\mathrm{t}|)=0.0011$ ). Similar results were found in the study conducted by Ismail $S$ Koltas et al.[8] and Agarawat et al.[9] In our study, majority of the patients (76\%) had MCV between $80-100 \mathrm{fL}$. MCV >100 was seen in total 7 cases. Similar results were found in the study conducted by Agarawat et al. In our study, reticulocyte count was more than $2 \%$ in $46 \%$ cases. Increased reticulocyte count was seen mostly in P. falciparum infections (54\%) compared to P. vivax infections (41.2\%). Similar results were found in the study conducted by Agarawat et al.[9] Present study shows elevated ESR in $82 \%$ of the cases. Similar observations were reported by Roy and Agaravat et al.[9] In the present study, leukocytosis was seen in $9 \%$ of the cases.
Double ' $\mathrm{t}$ ' test for correlation of total leukocyte count and \% parasitaemia was done and it was highly significant (Double ' $\mathrm{t}$ ' test $(|\mathrm{T}|>|\mathrm{t}|)=0.0076)$. Study by Bashawari et al.[10] show $7.2 \%$ leukocytosis. Leukopenia was seen in $19 \%$ of the total cases in present study. Bashwari et al. had leukopenia in $13.3 \%$ of the total malaria cases. Double ' $t$ ' test for correlation of monocyte and \% parasitaemia was also done and it was significant (Double ' $\mathrm{t}$ ' test $(|\mathrm{T}|>|\mathrm{t}|)=0.0376)$. In the present study, thrombocytopenia was seen in $88 \%$ of all malaria cases. Other studies like Richards et al. showed thrombocytopenia in $67 \%$ of the cases and Kueh et al. in $80 \%$ of the cases. In our study MPV values above $12 \mathrm{fL}$ were seen in 21 cases; $25.71 \%$ cases of falciparum showed MPV greater than $12 \mathrm{fL}$. Double ' $\mathrm{t}$ ' test for correlation of MPV and \% parasitaemia was done and it was highly significant (Double ' $\mathrm{t}$ ' test $(|\mathrm{T}|>|\mathrm{t}|)=0.0005$ ). Similar results were found in the study conducted by Facer CA et al.[7] Agarawat et al.[9] and Layla AM. In the present study, the degree of anaemia was correlated with the parasite load.

The Correlation Coefficient $(r)$ was $-0.50(\mathrm{P}<0.0001)$, which is highly significant and shows that decreased haemoglobin is one of the most common haematological parameter for the prediction of malaria cases and to assess the parasite load in malaria cases. Double ' $t$ ' test for correlation of haemoglobin and \% parasitaemia was done and it was highly significant (Double ' $t$ ' test $(|\mathrm{T}|>|\mathrm{t}|)=0.0010)$. Similarly, Sharma et al.[5] had anaemia which correlated with the parasite load. In the present study, degree of parasitaemia correlated with extent of thrombocytopenia. The Correlation Coefficient ( $r$ ) was-0.46 $(\mathrm{P}<0.0001)$, which is highly significant and shows that decreased platelet count is one of the most common haematological parameter for the prediction of malaria cases and to assess the parasite load in malaria cases. Double ' $t$ ' test for correlation of platelet count and \% parasitaemia was done and it was highly significant (Double ' $t$ ' test $(|\mathrm{T}|>|\mathrm{t}|)=0.0001$ ). Other studies like Ladhani et al.[11] show thrombocytopenia strongly associated with percentage parasitaemia.

\section{CONCLUSION}

In conclusion, the patient with acute intermittent febrile illness in the tropics with presence of changes in various haematological indices including degree of anaemia, RDW and thrombocytopenia usually increases the probability of malaria and correlates with parasite load. This may be used in addition to the clinical and microscopic parameters to heighten the suspicion of this disease with careful search for malaria parasite and prompt initiation of the therapy, thus limiting the mortality and prevent further complications.

\section{REFERENCES}

1. WHO. WHO expert committee on malaria: twentieth report 1998. Geneva, Switzerland 2000;p 71.

2. Erhart LM, Yingyuen K, Chuanak N, et al. Haematologic and clinical indices of malaria in a semi-immune population of western Thailand. Am J Trop Med Hyg 2004;70(1):8-14.

3. Jadhav UM, Patkar VS, Kadam NN. Thrombocytopenia in malaria-correlation with type and severity of malaria. J Assoc Physicians India 2004;52:615-8.

4. Svenson JE, MacLean JD, Gyrokos TW, et al. Imported malaria: clinical presentation and examination of symptomatic travelers. Archives of Internal Medicine 1995;155(8):861-8. 
5. Sharma SK, Das RK, Das BK, et al. Haematological and coagulation profile in acute falciparum malaria. J Assoc Physicians India 1992;40(9):581-3.

6. Biswas R, Sengupta G, Mundle M. A controlled study on haemograms of malaria patients in Calcutta. Indian J Malariol 1999;36(1-2):42-8.

7. Facer CA. Haematological aspects of malaria in: infection and haematology. Oxford: Butterworth Heinmann Ltd. 1994;pg 259-94.

8. Koltas IS, Demirhindi $\mathrm{H}$, Hazar $\mathrm{S}$, et al. Thrombocytopenia and red cell distribution width, supportive presumptive diagnosis of plasmodium vivax malaria. Saudi Med J 2007;28(4):535-9.
9. Agravat AH, Dhruva GA. Haematological changes in patients of malaria. Journal of Cell and Tissue Research 2010;10(3):2325-9.

10. Bashawri LAM, Mandil AA, Bahnassy AA, et al. Malaria: haematological aspects. Annals of Saudi Medicine 2002;22(5-6):372-7.

11. Ladhani S, Lowe B, Cole AO, et al. Changes in white blood cells and platelets in children with falciparum malaria: relationship to disease outcome. Brit J Haematol 2002;119(3):839-47. 